\title{
Nonasymptotic critical behavior from field theory
}

\author{
C. Bagnuls* and C. Bervillier ${ }^{\dagger}$ \\ C. E. Saclay, F91191 Gif-sur-Yvette Cedex, France
}

December 14, 2000

\begin{abstract}
The obtention (up to five or six loop orders) of nonasymptotic critical behavior, above and below Tc, from the field theoretical framework is presented and discussed.
\end{abstract}

When talking about critical behavior one usually thinks of critical exponents (power laws), and eventually of corrections to scaling, all notions strictly related to the unprecise definition of an asymptotic critical domain. In fact criticality may be observed beyond that theoretical domain and, sometimes, this makes it difficult to compare theory and experiments. [1] For example, it is thought that some systems could undergo a retarded crossover [2] from classical to Ising-like critical behaviors. In such a case, the critical domain would be much larger than for, say, pure fluids. Consequently many correctionto-scaling terms should be introduced and, it is very likely that the series would not converge. For that reason, nonasymptotic theoretical expressions of critical behaviors are required to describe such systems.

It is not very well known that, beyond the estimations of the critical exponents, the renormalization group ( $\mathrm{RG}$ ) theory [3] is also adapted to provide us with nonasymptotic forms of the critical behavior especially when a crossover phenomenon occurs (the crossover is then characterized by the competition of two fixed points).

We briefly present here the principles of the calculations done within the massive field theoretical framework in three dimensions $(d=3)$ [4] and which have yielded accurate nonasymptotic forms of the susceptibility $\chi(\tau)$ and the specific heat $C(\tau)$ for $\tau=(T-$ $\left.T_{c}\right) / T_{c}>0$ and $\tau<0$, of the correlation length $\xi(\tau)$ for $\tau>0$ and of the coexistence curve $M(\tau)$ for $\tau<0$. [5, 6] The calculations presented here have induced, directly or indirectly, several subsequent works. [77, 8] We hope that this text will encourage further works on nonasymptotic critical behavior. We think, in particular, that the variational perturbation theory used recently to estimate universal exponents [9] and amplitude ratios, [10] could be an advantageous tool.

Let us first specify the meaning of the title. "Nonasymptotic critical behavior" means that we perform a resummation of the infinite series of correction-to-scaling terms which are expected [1] in the asymptotic expression of any singular quantity such as $\xi(\tau)$. Particularly, for $\tau \rightarrow 0^{+,-}$, we have:

$$
\xi(\tau)=\xi_{0}^{+,-}|\tau|^{-\nu}\left[1+\sum_{n=1}^{\infty} \sum_{m=1}^{\infty} a_{\xi^{+,-}}^{(n, m)}|\tau|^{n \Delta_{m}}\right]
$$

*Service de Physique de l'Etat Condensé

†Service de Physique Théorique e-mail: bervil@spht.saclay.cea.fr 
in which $\nu$ is a critical exponent, $\xi_{0}^{+,-}$stands for the leading critical amplitudes in the two phases and the coefficients $a_{\xi^{+,-}}^{(n, m)}$ correspond to the amplitudes of the confluent corrections to scaling controlled by the exponents $\Delta_{m}(m=1,2, \cdots, \infty)$. Those exponents $(\nu$ and $\Delta_{m}$ ) arise in a linear study [11] of the RG transformation in the vicinity of a fixed point: the solutions of the eigenvalue problem provide us with some positive (say one, $\lambda_{0}$, for simplicity[) and infinitely many negative eigenvalues $\left(\lambda_{m}\right.$ for $\left.m=1,2, \cdots, \infty\right)$. Then we have $\nu=1 / \lambda_{0}, \Delta_{m}=-\nu \lambda_{m}(m=1,2, \cdots, \infty)$. The case $m=1$ corresponds to the first correction-to-scaling term associated to the largest negative eigenvalue and the usual notations are $\omega$ (for $-\lambda_{1}$ ) and $\Delta=\omega \nu$ (for $\Delta_{1}$ ). In a linear study of the RG, discarding the next-to-leading correction terms in Eq. (1), it is usual to write:

$$
\xi(\tau) \simeq \xi_{0}^{+,-}|\tau|^{-\nu}\left[1+a_{\xi}^{+,-}|\tau|^{\Delta}\right]
$$

with the universality of the ratios $\xi_{0}^{+} / \xi_{0}^{-}$and $a_{\xi}^{+} / a_{\xi}^{-}$. Eq. (2) is only valid asymptotically close to the critical point.

Notice that the infinite sum in Eq. (1) does not converge for large values of $\tau$. Thus, to get a useful nonasymptotic expression of the critical part of $\xi$, we must consider a resummation procedure. It is provided to us by the RG theory. However the framework we use implies an approximation: "from field theory" means that only one family of correction-to-scaling terms (associated to $m=1$ ) is accounted for. Thus, instead of Eq. (1), our effective expression for $\xi(\tau)$ is:

$$
\xi_{F T}(\tau)=\xi_{0}^{+,-}|\tau|^{-\nu}\left[1+\sum_{n=1}^{\infty} a_{\xi^{+,-}}^{(n)}|\tau|^{n \Delta}\right]
$$

As one could see from the use of our calculations in analyzing experimental [13] or MonteCarlo [14] data, the approximation of field theory does not prevent the study from yielding physically useful nonasymptotic critical behaviors. In fact, in the case of Eq. (3), the range $0<\tau<\infty$ corresponds to an interpolation between two fixed points and, consequently, the crossover is described by universal functions. [15]

\section{Principles of the calculations}

One starts from the "bare" or unrenormalized $\phi^{4}$-hamiltonian in $d$ euclidean dimensions corresponding to the scalar field theory to be renormalized:

$$
H=\int d^{d} x\left[\frac{1}{2}\left\{\left(\nabla \phi_{0}\right)^{2}+r_{0} \phi_{0}^{2}\right\}+\frac{g_{0}}{4 !} \phi_{0}^{4}\right]
$$

in which it is implicit that $\phi_{0}$ depends on $x$ and may eventually represents a vector with $n$ components in which case Eq. (雨) is supposed to satisfy the $O(n)$-symmetry. The bare coupling $g_{0}$ is dimensionful and "measured" in unit of $\Lambda$ (the ultra-violet cutoff):

$$
g_{0}=u_{0} \Lambda^{\epsilon}
$$

\footnotetext{
${ }^{1}$ The number of positive eigenvalues depends on the fixed point considered. Since we are interested in a critical point (we mean not a multi-critical point) then there is only one positive eigenvalue and the fixed point referred to in that case is the famous Wilson-Fisher [12] fixed point.
} 
in which $\epsilon=4-d$ and $u_{0}$ is dimensionless.

There exists two kinds of renormalization scheme for the scalar field theory: the massive and the Weinberg [16] schemes. In the massive scheme, the unit of reference is provided by the mass parameter $m=\xi^{-1}$. In this framework the critical theory, corresponding to $m=0$, is not defined. On the contrary, in the Weinberg scheme, one first defines the critical theory (massless theory) and the unit of length scale is provided by the inverse of some arbitrary momentum-subtraction-point parameter $\mu$. In that renormalization scheme, the "soft-mass" parameter $t$ is introduced via the renormalization of insertions of the $\phi_{0}^{2}$-operator within the vertex-functions; when it is different from zero, $t$ is (linearly) proportional to the reduced temperature scale $\tau$ defined above. Though we have effectuated our calculations within the massive framework — because the longest availablet perturbative series [17] have been obtained within the massive scheme directly in $d=3-$, the presentation of the principles of the calculation is simpler within the Weinberg scheme. In that scheme, the renormalization conditions correspond to the following (re)-definitions:

$$
\begin{aligned}
\phi_{0} & =\left[Z_{3}(u)\right]^{1 / 2} \phi \\
\left(\phi_{0}\right)^{2} & =\frac{Z_{3}(u)}{Z_{2}(u)}(\phi)^{2} \\
u_{0} \Lambda^{\epsilon} & =\mu^{\epsilon} u \frac{Z_{1}(u)}{\left[Z_{3}(u)\right]^{2}} \\
r_{0} & =r_{0 c}+\frac{Z_{2}(u)}{Z_{3}(u)} t
\end{aligned}
$$

in which $u$ is the renormalized coupling and $r_{0 c}$ is defined by:

$$
\left.\Gamma_{0}^{(0,2)}\left(p ; r_{0}, g_{0}\right)\right|_{p=0, r_{0}=r_{0 c}}=0
$$

in which the subscript 0 refers to the bare theory.

Up to analytical terms which are usually neglected when studying critical phenomena, the quantity $r_{0}-r_{0 c}$ is proportional to the physical parameter $\tau$ :

$$
\frac{r_{0}-r_{0 c}}{\Lambda^{2}}=\theta \tau+O\left(\tau^{2}\right)
$$

in which $\theta$ is a nonuniversal factor.

The renormalized $N$-point vertex-functions with $L$-insertions of the $(\phi)^{2}$ operator are related to their unrenormalized counter parts as follows:

$$
\Gamma^{(L, N)}(\{q, p\} ; u, \mu)=\left[Z_{3}(u)\right]^{N / 2}\left[\frac{Z_{2}(u)}{Z_{3}(u)}\right]^{L} \Gamma_{0}^{(L, N)}\left(\{q, p\} ; r_{0}, g_{0}\right)
$$

That the renormalization functions $Z_{i}(u)$ be defined by renormalization conditions on the 2-point and 4-point vertex-functions considered at some subtraction momentum point expressed in terms of the only dimensionful (momentum-like) parameter $\mu$ or by a "minimal" subtraction procedure (such as the subtraction of poles located at $\epsilon=0$ ) does not matter for the following.

\footnotetext{
${ }^{2}$ The unpublished Guelph report 17 may be obtained via H. Kleinert and V. Schulte-Frohlinde web site at http://www.physik.fu-berlin.de//kleinert/kleiner_reb8/programs/programs.html.
} 
In field theory, the RG originates from the arbitrariness of the subtraction procedure for a given bare theory. Hence, the renormalized quantities $u$ and $t$ become functions of the renormalization parameter $l=-\ln (\mu / \Lambda)$. Consequently, Eq. (8) must be understood as follows:

$$
r_{0}=r_{0 c}+\frac{Z_{2}[u(l)]}{Z_{3}[u(l)]} t(l)
$$

Now, by imposing that $t(l)$ remains a fixed quantity (say $t(l)=1$ ), one relates the evolution of $u(l)$ to the approach to the critical point of the bare (physical) theory (defined by $\left.r_{0} \rightarrow r_{0 c}\right)$. Then, with $t(l)=1$, Eq. (11) shows that, for $r_{0}=r_{0 c}, u(l)$ must take on a particular value $u^{*}$ so that $Z_{2}\left(u^{*}\right) / Z_{3}\left(u^{*}\right)$ vanishes. Of course, $u^{*}$ is the nontrivial zero of the famous $\beta$-function:

$$
\beta(u)=-\left.\frac{d u(l)}{d l}\right|_{u_{0}}
$$

with a positive $\omega=d \beta(u) /\left.d u\right|_{u=u^{*}}$ so that $u(l) \stackrel{l \rightarrow \infty}{\rightarrow} u^{*}$.

The pure scaling (power law) regime of vertex-functions corresponds to $u(l)=u^{*}$ and the first correction-to-scaling term [as in Eq. (2)] to a linear correction proportional to $u(l)-u^{*}$. As $u(l)$ moves further away from $u^{*}$, more and more correction terms must be included but then a nonlinear study is required. It is a matter of fact that the domain $0<u(l)<u^{*}$ corresponds to the entire domain $\infty>r_{0}-r_{0 c}>0$. Therefore, if one re-sums perturbative series in powers of $u(l)$ in the range $] 0, u^{*}[$, one implicitly obtains nonasymptotic critical answers which interpolate between a classical critical behavior (when $u(l)$ is small) and, say, an Ising-like critical behavior (for the $O(1)$-symmetry) when $u(l)$ approaches $u^{*}$. It remains to invert Eq. (11) to express these answers under the forms of functions of $r_{0}-r_{0 c}$ [or of $\tau$, via Eq. (10)] which is the genuine physical "measure" of the distance to the critical point.

With a view to get the best possible accuracy, we have looked at the available calculations up to relatively high orders of perturbation series. There are two kinds of such calculations:

1. analytically up to fifth order in the Weinberg scheme with dimensional regularization and minimal subtractions. 20]

2. numerically up to sixth order for $d=3$ in the massive scheme. [17

In the two cases, only the renormalization functions $Z_{i}$ are considered. This is because the theoretical interest is usually focused on the critical exponents, the obtention of which comes from the knowledge of the $Z_{i}$ 's. For example the series expansion for the critical exponent $\eta$ are given by:

$$
\eta(u)=\beta(u) \frac{d}{d u} \ln Z_{3}(u)
$$

once considered at $u=u^{*}$.

However, we are not simply interested in the critical exponents but in complete functions such as $\xi$ and $\chi$. Now, only in the case 2, the renormalization conditions are such that $\xi$ and $\chi$ are known in terms of the $Z_{i}$ 's. This is not true in the case 1 .

\footnotetext{
${ }^{3}$ We have also performed calculations $[18]$ for $u>u^{*}$. In this range, the sign of the first correctionto-scaling term is changed and corresponds to the Ising model [19].

${ }^{4}$ This is why the amplitude functions are known only up to three loop order in this scheme. [7]
} 
We denote the renormalized parameters of the massive scheme by $g$ and $m$ (instead of $u$ and $t$ ). Their relations to the bare parameters are similar to those given by Eqs. (5) 9) except that in addition to the change $u \rightarrow g$, Eqs. (78) now read:

$$
\begin{aligned}
& g_{0}=m^{\epsilon} g \frac{Z_{1}(g)}{\left[Z_{3}(g)\right]^{2}} \\
& r_{0}=\delta m^{2}+\frac{m^{2}}{Z_{3}(g)}
\end{aligned}
$$

The mass shift $\delta m^{2}$ is defined by a subtraction condition which avoids the explicit consideration of $r_{0 c}$ via Eq. (9), namely:

$$
\Gamma^{(0,2)}(0 ; m, g)=m^{2}
$$

The other subtraction conditions 5 wich define the $Z_{i}$ 's read:

$$
\begin{aligned}
\left.\frac{d}{d p^{2}} \Gamma^{(0,2)}(p ; m, g)\right|_{p=0} & =1 \\
\Gamma^{(0,4)}(\{0\} ; m, g) & =m^{\epsilon} g \\
\Gamma^{(1,2)}(\{0,0\} ; m, g) & =1
\end{aligned}
$$

so that the physical (bare) quantities $\xi$ and $\chi$ are given by:

$$
\begin{aligned}
& \xi^{-1}(g)=m=g_{0} \frac{\left[Z_{3}(g)\right]^{2}}{g Z_{1}(g)} \\
& \chi^{-1}(g)=Z_{3}^{-1} m^{2}=g_{0}^{2} \frac{\left[Z_{3}(g)\right]^{3}}{\left[g Z_{1}(g)\right]^{2}}
\end{aligned}
$$

The re-summations of the perturbative series for those quantities have been done using the technique initiated by Le Guillou and Zinn-Justin [21] after having accounted for the singularities of the $Z_{i}$ 's at the fixed point $g^{*}$. They may be easily treated by writing, e.g. for $Z_{3}(g)$ which has a singularity at $g^{*}$ of the form $\left(g^{*}-g\right)^{\eta / \omega}$ :

$$
Z_{3}(g)=Z_{3}(y) \exp \left\{\int_{y}^{g} \frac{\eta(x)}{\beta(x)} d x\right\}
$$

in which $y$ is some small value of $g$, the definitions of $\beta(x)$ and $\eta(x)$ being unchanged in their forms compared to Eqs. (12, 13). Let us mention that some difficulties could be encountered in the resummation procedure due to nonanalytic confluent singularities [22] in the $\beta$-function at $g^{*}$, but to date they have not been numerically observed.

Thus, in the homogeneous phase, the physical quantities $\xi$ and $\chi$ can be easily estimated as functions of $g$ in the range ]0, $g^{*}$ [ from the calculated series. [17] However our aim was to obtain those quantities as function of $\tau$ (i.e. of $r_{0}-r_{0 c}$ ). Now, the massive framework uses $m \propto \tau^{\nu}$ instead of $t \propto \tau$, therefore the linear relation to $\tau$ is lost. In order to reintroduce it, we use the fact that, at zero-external-momenta:

\footnotetext{
${ }^{5}$ Which eliminates the quadratic ultra-violet divergences occuring at $d=4$.

${ }^{6}$ Which eliminate the logarithmic ultra-violet divergences occuring at $d=4$.
} 


$$
\left[Z_{2}(g)\right]^{-1}=\Gamma_{0}^{(1,2)}\left(r_{0}, g_{0}\right)=\left.\frac{\partial}{\partial r_{0}} \Gamma_{0}^{(0,2)}\left(r_{0}, g_{0}\right)\right|_{g_{0}}
$$

Using Eqs (14 16), we reexpress this under the following form:

$$
\frac{d\left(r_{0} / g_{0}^{2}\right)}{d g}=Z_{2}(g) \frac{d}{d g}\left\{\frac{\left[Z_{3}(g)\right]^{3}}{\left[g Z_{1}(g)\right]^{2}}\right\}
$$

which, after integration allows us to (implicitly) define an effective (and nonperturbative) critical value $r_{0 c}^{\prime}$ by reference to the fixed point value $g^{*}$ :

$$
\tilde{t}(g) \equiv \frac{r_{0}-r_{0 c}^{\prime}}{g_{0}^{2}}=-\int_{g}^{g^{*}} d x Z_{2}(x) \frac{d}{d x}\left\{\frac{\left[Z_{3}(x)\right]^{3}}{\left[x Z_{1}(x)\right]^{2}}\right\}
$$

The integrand of Eq. (17) may be estimated using the same procedure as before and the integration has been done numerically yielding the numerical evolution of $\tilde{t}(g)$ in the range $] 0, g^{*}$. The final results (the functions $\xi(\tilde{t})$ and $\chi(\tilde{t})$ ) were obtained after a fitting procedure of the implicit form $\xi(g), \chi(g)$ and $\tilde{t}(g)$. This summarizes the calculations done in the homogeneous phase [5] which included also the specific heat $C(\tilde{t})$ the perturbative series of which were previously [23] extracted from the Guelph report; [17] the calculations were performed for the symmetries $n=1,2$ and 3 .

\section{Calculations in the inhomogeneous phase and the critical bare mass}

"...it is more difficult to calculate physical quantities in the ordered phase because the theory is parametrized in terms of the disordered phase correlation length $m^{-1}$ which is singular at $T_{c}$. Also the normalization of correlation functions is singular at $T_{c}$." [24]

We have calculated [6] the perturbative series for the free energy directly at $d=3$ using the numerous already-estimated Feynman integrals of the massive scheme [17 and new kinds of integrals which have been estimated for the occasion.

Because the free energy is generally written in terms of $T-T_{c}$, we have been led to explicitly consider the delicate question of the critical bare mass. Indeed, it is known that the perturbative series of super-renormalizable massless field theories (such as $\phi_{d<4}^{4}$ ) develop infrared singularities which are usually simply ignored within the $\epsilon$-expansion framework. In 1973, using a dimensional regularization, Symanzik [25] has shown that the critical bare mass - which has the form $r_{0 c}=g_{0}^{2 / \epsilon} f(\epsilon)$ in which $f(\epsilon)$ has poles at $\epsilon=2 / k(k=1,2, \ldots \infty)$ - is in fact an infrared regulator for the theory. However the final result (free of infrared divergences) is no longer perturbative (e.g., logarithms of $g_{0}$ appear at $d=3)$. Though the nonperturbative nature of $r_{0 c}$ is an important aspect of the RG theory, [26] this question may be circumvented when looking at the critical behavior. This is because $T_{c}$ is a nonuniversal quantity, thus its explicit determination is not required, only the difference $T-T_{c}$ is needed. Hence, provided that Eq. (9) be again satisfied, one may redefine $r_{0 c}$ [as it has been done in Eq. ([17)]. Consequently, it is allowed [6] to perform a particular mass-shift $r_{0}=r_{0}^{\prime}+\delta r_{0}(\epsilon)$ in such a way that $\delta r_{0}(\epsilon)$ subtracts the poles occuring at $\epsilon=1$, and to fix afterwards the critical temperature in terms of $r_{0}^{\prime}$. 
The series for the free energy have then been obtained graph by graph up to five loops according to the following rules:

1. graphs involving only $\phi^{4}$-vertices which were already estimated [17] with the massshift parameter $\delta m^{2}$ [defined by Eq. (15, 16)] have been re-expressed to account for the mass-shift parameter $\delta r_{0}(\epsilon)$.

2. New Feynman integrals at $d=3$ with their weights involving:

- exclusively $\phi^{3}$-vertices have been calculated and compared to existing estimates. [27]

- $\phi^{3}$-vertices mixed with a single $\phi^{4}$-vertex have been estimated for the first time for the occasion.

Those series for the free energy have been used by Guida and Zinn-Justin [8] to give an accurate estimation of the scaled equation of state. But this kind of consideration does not account for any correction-to-scaling terms and the comparison with experiments is not easy. Instead, we were again interested in actually measurable quantities like the susceptibility $\chi$, the specific heat $C$ and the spontaneous magnetization $M$ in the inhomogeneous phase. We have not considered the correlation length $\xi$ in this phase because the required Feynman integrals were not calculated by Nickel et al; [17] this quantity has been considered afterwards at $d=3$ but up to 3-loop order only. [28]

Because the renormalization procedure is unchanged in going into the broken-symmetry phase, the critical singularities at the fixed point $g^{*}$ may be taken into account with the same renormalization functions $Z_{i}(g)$ as defined previously. But the relation between the linear measure of the distance to $T_{c}$ and the unchanged renormalized coupling $g$ is different. Instead of Eq. (17) we have obtained:

$$
\tilde{t}^{\prime}(g)=-\int_{g}^{g^{*}} d x\left\{Z_{2}(x) \frac{d}{d x}\left\{\frac{\left[Z_{3}(x)\right]^{3}}{\left[x Z_{1}(x)\right]^{2}}\right\}[1-U(x)]\right\}
$$

in which $U(g)$ is given [6] as power series in $g$.

Obviously, our nonasymptotic study of the critical behavior accounts for all the universal properties which are expected when $\tau \rightarrow 0$. Consequently as a by-product, estimates of universal combinations of leading critical amplitudes were given for the first time from the five loop order at $d=3$. The recent careful re-estimations [8, 29] of those universal combinations from the same series have mainly reduced the error-bars. We also gave for the first time accurate estimates of some [30] of the universal ratios of the first confluent correction-to-scaling [6].

\section{References}

[1] H. Güttinger and D. S. Cannell, Phys. Rev. A24, 3188 (1981).

[2] Y. Levin and M. E. Fisher, Physica A225, 164 (1996). N. V. Brilliantov, C. Bagnuls and C. Bervillier, Phys. Lett. A245, 274 (1998). C. Bagnuls and C. Bervillier, Cond. Matt. Phys. 3, 559 (2000). 
[3] K. G. Wilson and J. Kogut, Phys. Rep. 12C, 77 (1974).

[4] G. Parisi, J. Stat. Phys. 23, 49 (1980).

[5] C. Bagnuls and C. Bervillier, Phys. Rev. B32, 7209 (1985); J. Physique Lett. 45, L95 (1984).

[6] C. Bagnuls, C. Bervillier, D. I. Meiron and B. G. Nickel, Phys. Rev. B35, 3585 (1987). See also "Addendum-erratum to: "Nonasymptotic critical behavior from field theory at $d=3$. II. The ordered-phase case" Phys. Rev. B35, 3585 (1987).", hep-th/0006187 (2000).

[7] R. Schloms and V. Dohm, Nucl. Phys. B328, 639 (1989); Phys. Rev. B42, 6142 (1990). H. J. Krause, R. Schloms and V. Dohm, Z. Phys. B79, 287 (1990). F. J. Halfkann and V. Dohm, Z. Phys. B89, 79 (1992). S. S. C. Burnett, M. Strösser and V. Dohm, Nucl. Phys. B504, 665 (1997); Nucl. Phys. B509, 729(E) (1998). M. Strösser, S. A. Larin and V. Dohm, Nucl. Phys. B540, 654 (1999). M. Strösser, M. Mönnigmann and V. Dohm, Physica B284, 41 (2000).

[8] R. Guida and J. Zinn-Justin, Nucl. Phys. B489, 626 (1997).

[9] H. Kleinert, Phys. Lett. B434, 74 (1998); Phys. Lett. B463, 69 (1999); Phys. Rev. D57, 2264 (1998); Addendum: Phys. Rev. D58, 107702 (1998).

[10] H. Kleinert and B. Van den Bossche, cond-mat/0011329 (2000).

[11] F. J. Wegner, Phys. Rev. B5, 4529 (1972) and in Phase Transitions and Critical Phenomena Vol. VI, p. 7, Ed. by C. Domb and M.S. Green (Acad. Press, N.-Y., 1976).

[12] K. G. Wilson and M. E. Fisher, Phys. Rev. Lett. 28, 240 (1972).

[13] C. Bagnuls and C. Bervillier, Phys. Rev. Lett. 58, 435 (1987). C. Bagnuls, C. Bervillier and Y. Garrabos, J. Physique Lett. 45, L127 (1984). C. W. Garland, G. Nounesis, M. J. Young and R. J. Birgeneau, Phys. Rev. E47, 1918 (1993).

[14] K. Binder and E. Luijten, Comp. Phys. Comm. 127, 126 (2000).

[15] See also: A. Pelissetto, P. Rossi and E. Vicari, Phys. Rev. E58, 7146 (1998); S. Caracciolo, M. S. Causo, A. Pelissetto, P. Rossi and E. Vicari, Nucl. Phys. B (Proc. Suppl.) 73, 757 (1999); and the recent review: A. Pelissetto and E. Vicari, condmat/0012164 (2000). On the crossover phenomenon in fluids and fluid mixtures, see for example: M. A. Anisimov and J. V. Sengers, "Critical region", in "Equations of State for Fluids and Fluid Mixtures", p. 381, Ed. by J. V. Sengers, R. F. Kayser, C. J. Peters and H. J. White Jr (Elsevier, Amsterdam, 2000). See also the discussion: C. Bagnuls and C. Bervillier, Phys. Rev. Lett. 76, 4094 (1996); M. A. Anisimov, A. A. Povodyrev, V. D. Kulikov and J. V. Sengers, ibid, 4095.

[16] S. Weinberg, Phys. Rev. D8, 3497 (1973).

[17] B. G. Nickel, D. I. Meiron and G. A. Baker, Jr, "Compilation of 2-pt and 4-pt graphs for continuous spin models", Gelph University preprint, unpublished (1977). 
[18] C. Bagnuls and C. Bervillier, Phys. Lett. A195, 163 (1994).

[19] C. Bagnuls and C. Bervillier, Phys. Rev. B41, 402 (1990). A. Liu and M. E. Fisher, J. Stat. Phys. 58, 431 (1990). B. G. Nickel and B. Sharpe, J. Phys. A12, 1819 (1979).

[20] K. G. Chetyrkhin, A. L. Kataev and F. V. Tkachov, Phys. Lett. 99B, 147 (1981); Phys. Lett. 101B, 457 (1981). K. G. Chetyrkhin, S. G. Gorishny, S. A. Larin and F. V. Tkachov, Phys. Lett. 132B, 351 (1983). S. G. Gorishny, S. A. Larin and F. V. Tkachov, Phys. Lett. 101A, 120 (1984). H. Kleinert, J. Neu, V. Schulte-Frohlinde, K. G. Chetyrkhin and S. A. Larin, Phys. Lett. B272, 39 (1991); Erratum, Phys. Lett. B319, 545 (1993).

[21] J. C. Le Guillou and J. Zinn-Justin, Phys. Rev. Lett. 39, 95 (1977).

[22] B. G. Nickel, in "Phase Transitions", p. 291, Ed. by M. Lévy, J.C. Le Guillou and J. Zinn-Justin (Plenum Press, N.Y. and London, 1982). C. Bagnuls and C. Bervillier, J. Phys. Stud. 1, 366 (1997). A. Pelissetto and E. Vicari, Nucl. Phys. B (Proc. Suppl.) 73, 775 (1999). M. Caselle, A. Pelissetto and E. Vicari, hep-th/0010228 (2000).

[23] C. Bervillier and C. Godrèche, Phys. Rev. B21, 5427 (1980).

[24] J. Zinn-Justin, in "Euclidean Field Theory and Critical Phenomena", "First edition" (Oxford University Press, 1989), p. 614.

[25] K. Symanzik, Lett. Nuovo Cimento 8, 771 (1973). See also: G. Parisi, Nucl. Phys. B150, 163 (1979). R. Jackiw and S. Templeton, Phys. Rev. D23, 2291 (1981). M. C. Bergère and F. David, Ann. Phys. (N.Y.) 142, 416 (1982).

[26] C. Bagnuls and C. Bervillier, Phys. Rev. Lett. 60, 1464 (1988).

[27] J. Reeve, A. J. Guttmann and B. Keck, Phys. Rev. B26, 3923 (1982). J. Reeve, J. Phys. A15, L521 (1982).

[28] C. Gutsfeld, J. Küster and G. Münster, Nucl. Phys. B479, 654 (1996).

[29] R. Guida and J. Zinn-Justin, J. Phys. A31, 8103 (1998).

[30] See also: C. Bagnuls and C. Bervillier, Phys. Rev. B24, 1226 (1981). 